\title{
CHARACTERIZATIONS OF MAXIMAL TOPOLOGIES
}

\author{
JAMES E. JOSEPH
}

(Received 31 January 1975; revised 3 April 1975)

\begin{abstract}
New characterizations of maximal topologies are given for a class of topologies including the compact, Lindelöf and $m$-compact topologies.

In what follows, let $\lambda$ represent a property of topological spaces. If a space $(X, T)$ has property $\lambda$, we will say that $T$ is a $\lambda$-topology on $X$ and call the space a $\lambda$-space. For certain choices of $\lambda$, characterizations have been given for maximal elements in the set of $\lambda$-topologies ordered by inclusion. For example, the following has been proved where $\lambda$ is replaced by either of the words, "compact," "Lindelöf," or "countably compact," Joseph (1969), Raha (1973), Smythe and Wilkins (1963).
\end{abstract}

THEOREM. The following statements are equivalent for a space $(X, T)$.

1) $T$ is a maximal $\lambda$-topology on $X$.

2) The set of $\lambda$-subspaces of $X=$ the set of closed subsets of $X$.

3) Any continuous bijection from a $\lambda$-space to $X$ is a homeomorphism.

Recently, the following result has been obtained, Joseph (to appear):

THEOREM. The following statements are equivalent to the statements in the above theorem with $\lambda$ replaced by "compact."

4) Any continuous surjection from a compact space to $X$ is a closed quotient map.

5) Any function with a compact graph from $X$ to a space is continuous.

6) Any function with a compact graph from a space to $X$ is closed when $T$ is a compact topology.

We note that the following statements are true of a $\lambda$-space $(X, T)$ with $\lambda$ replaced throughout by either "compact," "Lindelöf," "countably compact," 
or " $m$-compact" for an infinite cardinal, $m$. These properties are readily verified by arguments paralleling those in Raha (1973).

(*) 1) A closed subspace of $X$ is a $\lambda$-space.

2) A continuous image of $X$ is a $\lambda$-space.

3) For each $\lambda$-subspace, $A$ of $X$, the supremum, $Q(T, A)$, of $T$ and $\{X, X-A, \varnothing\}$ is a $\lambda$-space.

In this paper, we prove the following:

THEOREM. If $\lambda$ is a property of topological spaces such that any $\lambda$-space satisfies the properties listed in $\left(^{*}\right)$ the following statements are equivalent for a space $(X, T)$.

1) $T$ is a maximal $\lambda$-topology on the set $X$.

2) The set of $\lambda$-subspaces of $X=$ the set of closed subsets of $X$.

3) Any continuous surjection from a $\lambda$-space to $X$ is a closed quotient map.

4) Any continuous bijection from a $\lambda$-space to $X$ is a homeomorphism.

5) Any function with a $\lambda$-graph from $X$ is continuous.

6) Any function with a $\lambda$-graph into $X$ is closed when $T$ is a $\lambda$-topology on $X$.

Proof. Let $\pi_{x}$ and $\pi_{y}$ be the projections of $X \times Y$ onto $X$ and $Y$ respectively. To show that 1) implies 2) we need show only that each $\lambda$-subspace of $X$ is closed. This is clear since for each $\lambda$-subspace, $A$, of $X, T \subset Q(T, A)$ and $Q(T, A)$ is a $\lambda$-topology on $X$ forcing $T=Q(T, A)$. Assuming 2), let $Y$ be a $\lambda$-space and let $g: Y \rightarrow X$ be a continuous surjection. If $A$ is closed in $Y$, then $A$ is a $\lambda$-subspace of $Y$, so $g(A)$ is a $\lambda$-subspace of $X$ and thus is closed in $X$. Now, let $T^{*}$ be any topology on $X$ for which $g$ is continuous and $T \subset T^{*}$. Then $X=g(Y)$, so $\left(X, T^{*}\right)$ is a $\lambda$-space. Let $A$ be $T^{*}$-closed in $X$. Then $A$ is a $T^{*}-\lambda$-subspace of $X$, so $A$ is a $T$ - $\lambda$-subspace of $X$ since the identity function from $\left(X, T^{*}\right)$ to $(X, T)$ is continuous. So, $A$ is $T$-closed, $T=T^{*}$, and $g$ is a quotient map. It is immediate that 3 ) implies 4). To show that 5) follows from 4), let $Y$ be a space and suppose $g: X \rightarrow Y$ has a $\lambda$-graph, $G(g)$. The restriction, $\pi_{x}^{*}$, of $\pi_{x}$ to $G(g)$ is a continuous bijection and thus is a homeomorphism. Since $g=\pi_{y} \circ \pi_{x}^{*-1}, g$ is continuous. To prove that 5) implies 6) let $g: Y \rightarrow X$ be a function with $G(g)$ a $\lambda$-subset of $Y \times X$ and let $A \subset Y$ be closed. Then for the restriction, $\pi_{y}^{*}$, we have $\pi_{y}^{*-1}(A)$ is closed in $G(g)$ and thus is a $\lambda$-subspace of $Y \times X$. Then $g(A)=\pi_{x}\left(\pi_{y}^{*-1}(A)\right)$ is a $\lambda$-subspace in $X$. Since $X$ is a $\lambda$-space, the identity function, $i$, from $(X, T)$ to $(X, Q(T, g(A)))$ has a $\lambda$-graph because the function, $h$, from $(X, T)$ to $X \times X$ defined by $h(x)=(x, x)$ is continuous $(T \subset Q(T, g(A)), \quad Q(T, g(A)) \subset$ $Q(T, g(A))$ and $h(X)=G(i))$; so $i$ is continuous. Thus $g(A)$ is $T$-closed since $g(A)$ is $Q(T, g(A))$-closed. Finally, to verify that 6) implies 1), let $T^{*}$ be a $\lambda$-topology on $X$ and suppose $T \subset T^{*}$. The identity function from $\left(X, T^{*}\right)$ to 
$(X, T)$ renders $T$ a $\lambda$-topology and, with the same reasoning as above, has a $\lambda$-graph; and is thus closed. Therefore, $T=T^{*}$. This completes the proof.

The theorem is true when $\lambda$ is replaced throughout by either of the words "compact," "Lindelöf," or " $m$-compact" for any infinite cardinal $m$.

Using the result of the theorem for $m$-compactness and the known fact that $m$-compact subsets are closed in a Hausdorff topological space with an open base of cardinality $\leqq m$ at each point, we may prove the following generalizations of corollaries 5 and 6 in Raha (1973).

COROLLARY 1. If $T$ is an m-compact Hausdorff topology which contains an open base of cardinality $\leqq m$ at each point, then $T$ is maximal $m$-compact.

COROLlARY 2. If T is an m-compact Hausdorf topology which contains an open base of cardinality $\leqq m$ at each point, then $T$ is a minimal element in the set of Hausdorff topologies which contain an open base of cardinality $\leqq m$ at each point.

Proof. Any topology which is contained in $T$ would be $m$-compact; if in addition, the topology is Hausdorff and contains an open base of cardinality $\leqq m$ at each point, the topology must be maximal $m$-compact. Since $T$ is $m$-compact, this topology must be $T$. This completes the proof.

\section{References}

J. Joseph (1969), 'Continuous functions and spaces in which compact sets are closed', Amer. Math. Monthly 76, 1125-1126.

J. Joseph (to appear), 'Compact graphs and spaces in which compact sets are closed', Math. Mag.

A. B. Raha (1973), 'Maximal topologies', J. Austral. Math. Soc. 15, 279-290.

N. Smythe and C. A. Wilkins (1963), 'Minimal Hausdorff and maximal compact spaces', J. Austral. Math. Soc. 3, 167-171.

The Federal City College

Washington, D. C.

U.S.A. 